\title{
N $91-32658$
}

\section{HORIZONTAL FIELDS GENERATED BY RETURN STROKES}

\author{
Vernon Cooray \\ Institute of High Voltage Research \\ (A department at Uppsala University) \\ Uppsala, Sweden
}

\begin{abstract}
Horizontal fields generated by return strokes play an important role in the interaction of lightning generated electromagnetic fields with overhead power lines. In many of the recent investigations on the interaction of lightning electromagnetic fields with power lines, the horizontal field was calculated by employing the expression for the tilt of the electric field of a plane wave propagating over finitely conducting earth. In this paper we show that the horizontal field generated by return strokes over finitely conducting ground can be obtained to a high accuracy by using the expression for the surface impedance of the finitely conducting earth. The method is suitable to calculate horizontal fields generated by return strokes at distances as close as $200 \mathrm{~m}$. At these close ranges the use of the wavetilt expression can cause large errors.
\end{abstract}

\section{INTRODUCTION}

Recent experimental investigations indicate that the electric field component parallel to the ground plane i.e. horizontal field, plays a dominant role in the interaction of lightning generated electromagnetic fields with over head power lines $[1,2,3]$. The accuracy of the induced voltage calculations depends, therefore, on the accuracy to which the horizontal field generated by lightning return strokes can be calculated. These calculations require the knowledge concerning the electromagnetic fields generated by dipoles over finitely conducting earth. The pioneering work on the electromagnetic fields generated by dipoles over finitely conducting ground was done by Sommerfeld [4]. He presented the solutions to the problem in integral form. The horizontal fields generated by lightning return strokes over finitely conducting earth can be written in terms of these integrals and the results can be obtained by solving these equations numerically. However, this method of calculation is not practical because of the limitations on the computational time. To overcome these problems many researchers have used different approximations to calculate the horizontal fields generated by lightning return strokes.

One of the approximations used frequently in the literature, to calculate the horizontal fields generated by lightning, is the method of "Wavetilt". When a plane electromagnetic wave propagates over finitely conducting ground the electric field at the surface is tilted towards the ground (in the case of perfectly conducting earth the electric field is perpendicular to the earth's surface). That is, at the surface of the ground the electric field component parallel to the ground surface is not zero. The magnitude of the tilt, and hence the amplitude of the horizontal electric field, depends on the conductivity and dielectric constant of the ground. According to the wavetilt expression the ratio between the electric field component perpendicular to the ground, $\mathrm{Ez}$, and the electric field component parallel to the ground, Eh, in frequency domain is given by

$E_{h}(j \omega) / E_{z}(j \omega)=1 /\left(\varepsilon+\sigma / j \omega \varepsilon_{0}\right)^{0.5}$

where $\varepsilon$ is the relative dielectric constant, $\sigma$ is the conductivity, $\varepsilon_{0}$ is the permittivity of free space and $\omega$ is the angular frequency. In most of the studies mentioned earlier the above 
relationship was used to calculate the horizontal filed from either the measured or the calculated vertical electric field. However, the above relationship is valid under two conditions, namely, (a) the fields are radiation and (b) the angle of incidence is near grazing. Unfortunately, very close to the lightning return stroke channel, both these conditions could be violated and the horizontal field calculated from the wavetilt expression could be in error. On the other hand, the horizontal field can be related to the horizontal magnetic field, $B_{h}(j \omega)$, through the expression for the surface impedance of the earth ( the earth is assumed to be flat and homogeneous) as follows :

$E_{h}(j \omega) / B_{h}(j \omega)=c /\left(\varepsilon+\sigma / j \omega \varepsilon_{0}\right)^{0.5}$

where $c$ is the speed of light in free space. In this paper we will show that this relationship can be used to calculate the horizontal fields generated by lightning at close ranges to a higher accuracy than is possible from the wavetilt formula. We will also investigate the errors caused by the use of wavetilt expression to calculate horizontal fields when the lightning return strokes are within about $2 \mathrm{~km}$ from the point of observation.

\section{FIELDS GENERATED BY A VERTICAL DIPOLE OVER FINITELY CONDUCTING EARTH}

As we have mentioned earlier many approximate solutions are available for the fields generated by vertical dipoles over finitely conducting ground $[5,6,7,8]$. In this paper we will consider the solutions given by Bannister [8]. Recently these solutions were compared with the exact integrations of Sommerfeld integrals by Zaddam and Degauque [9]. Their results show that the equations given by Bannister [8] can be used to calculate fields to an accuracy higher than $5 \%$.

Let us consider the geometry shown in figure 1 . The dipole is at a height $h$ from ground level and the current in the dipole is directed in the direction of $z$ axis. The ground is assumed to be flat and homogeneous and is associated with the constants $\varepsilon$ and $\sigma$. According to Bannister [8] the electromagnetic field components at point $P$ are given by (see figure 1 and the 'glossary of symbols' for the definition of parameters used in these equations)

$$
\begin{aligned}
E_{h}(j \omega) & =\frac{M}{4 \pi \varepsilon_{0} j \omega}\left\{\left(3+3 \gamma_{0} R_{0}+\gamma_{0}^{2} R_{0}^{2}\right) \sin \psi_{0} \cos \psi_{0} \frac{e^{-\gamma_{0} R_{0}}}{R_{0}{ }^{3}}\right. \\
+ & \left(3+3 \gamma_{0} R_{1}+\rho \gamma_{0}^{2} R_{1}^{2}\right) \sin \psi_{1} \cos \psi_{1} \frac{e^{-\gamma_{0} R_{1}}}{R_{1}{ }^{3}} \\
& -\frac{2}{n^{2}}\left(3+3 \gamma_{0} R_{1}+\gamma_{0}^{2} R_{1}^{2}\right) \sin \psi_{1} \cos \psi_{1} \frac{e^{-\gamma_{0} R_{1}}}{R_{1}^{3}} \\
+ & \gamma_{0}^{2}\left[\frac{\cos \psi_{2} e^{-\gamma_{0} R_{2}}}{R_{2}+d+z+h}-\frac{\cos \psi_{1} e^{-\gamma_{0} R_{1}}}{R_{1}+z+h}\left(1+\gamma_{0} d\right)\right] \\
+ & \left.2 \Delta\left[1-\left(\frac{1-\rho}{2}\right) F(\omega)\right] \cos \psi_{1} \gamma_{0}^{2} R_{1}{ }^{2} \frac{e^{-\gamma_{0} R_{1}}}{R_{1}^{3}}\right\}
\end{aligned}
$$




$$
\begin{aligned}
E_{z}(j \omega)= & -\frac{M}{4 \pi \varepsilon_{0} j \omega}\left\{\left[\left(1-3 \sin ^{2} \psi_{0}\right)\left(1+\gamma_{0} R_{0}\right)+\gamma_{0}^{2} R_{0}^{2} \cos ^{2} \psi_{0}\right] \frac{e^{-\gamma_{0} R_{0}}}{R_{0}{ }^{3}}\right. \\
+ & {\left[\left(1-3 \sin ^{2} \Psi_{1}\right)\left(1+\gamma_{0} R_{1}\right)+\rho \gamma_{0}^{2} R_{1}^{2} \cos ^{2} \psi_{1}\right] \frac{e^{-\gamma_{0} R_{1}}}{R_{1}{ }^{3}} } \\
+ & \left.(1-\rho) F(\omega) \cos ^{2} \Psi_{1}\left(\gamma_{0}^{2} R_{1}^{2}\right) \frac{e^{-\gamma_{0} R_{1}}}{R_{1}{ }^{3}}\right\} \\
B_{p}(j \omega)= & \frac{M \mu_{0}}{4 \pi}\left\{\left(1+\gamma_{0} R_{0}\right) \cos \Psi_{0} \frac{e^{-\gamma_{0} R_{0}}}{R_{0}^{2}+\left(1+\rho \gamma_{0} R_{1}\right) \cos \Psi_{1} \frac{e^{-\gamma_{0} R_{1}}}{R_{1}^{2}}}\right. \\
& \left.+(1-\rho) F(\omega) \cos \psi_{1}\left(\gamma_{0} R_{1}\right) \frac{e^{-\gamma_{0} R_{1}}}{R_{1}^{2}}\right\}
\end{aligned}
$$

According to Bannister [8] these equations are valid at any point on the earth provided that $\mathrm{n}^{2}>1$. Now, the dominant angular frequencies in the lightning generated electromagnetic fields lie in the range of $10^{4}$ to $10^{7} \mathrm{rad} / \mathrm{s}$ and the conductivity and the dielectric constant of typical soils are distributed in the range of 0.01 to $0.001 \mathrm{~S} / \mathrm{m}$ and 3 to 10 respectively. Calculations show that $n^{2} \gg 1$ for the values of frequencies, conductivity and dielectric constants in the above ranges. Therefore we conclude that the above equations could be used to calculate the fields generated by lightning over finitely conducting ground.

\section{COMPARISON OF THE HORIZONTAL FIELDS CALCULATED FROM WAVETILT AND SURFACE IMPEDANCE WITH THE EXACT EXPRESSION}

Let $\mathrm{E}_{\mathrm{hw}}(\mathrm{j} \omega)$ represents the horizontal field at ground level as calculated by using the wavetilt expression (i.e. equation 1 ), $\mathrm{E}_{\mathrm{hs}}(\mathrm{j} \omega)$ represents the horizontal field at ground level as calculated by using the surface impedance expression (i.e. equation 2 ) and $E_{h}(j \omega)$ represents the exact value of the horizontal field at ground level as given by equation (3). We have calculated these field components over a large range of frequencies, heights of the dipole and distances. From these calculations we have estimated the ratio $\mid E_{h w} / E_{h} l$ and $\mid E_{h s} / E_{h} l$. The variation of these ratios as a function of distance, conductivity, height of the dipole from ground level and frequency are shown in figures 2 through 4 . In each figure the solid line represents the ratio $\left|E_{h s} / E_{h}\right|$ and the dashed line represents the ratio $\left|E_{h w} / E_{h}\right|$. Figure 2 shows how these ratios vary as a function of frequency and conductivity when the dipole is at ground level. Figures 3 and 4 correspond to dipoles at heights $500 \mathrm{~m}$ and $1000 \mathrm{~m}$ respectively.

Let us consider the results in figure 2. These results show that for distances greater than about $200 \mathrm{~m}$ from the dipole the ratio $\left|\mathrm{E}_{\mathrm{hs}} / \mathrm{E}_{\mathrm{h}}\right|$ is equal to unity. Figures 3 and 4 show that this is also 
true for dipoles at heights $500 \mathrm{~m}$ and $1000 \mathrm{~m}$ from ground level. That is for distances greater than $200 \mathrm{~m}$ and for dipoles situated at heights between 0 to $1000 \mathrm{~m}$ the horizontal field calculated by using the surface impedance expression is an accurate representation of the horizontal field component at ground level i.e. $E_{h s}(j \omega)=E_{h}(j \omega)$.

On the other hand the ratio $\mid \mathrm{E}_{\mathrm{hw}} / \mathrm{E}_{\mathrm{h}} \mathrm{l}$ is far from unity, specially at small distances and low frequencies. However, with increasing distance and frequency the results obtained from the wavetilt expression approaches that obtained by using the exact expression. The reason for this is that with increasing distance the radiation field becomes the dominant component in the electric field, which enhance the validity of the wavetilt expression. However, as one can see from these figures, the horizontal fields calculated by using the wavetilt expression would be in error even at distances as large as $5000 \mathrm{~m}$ when the frequencies of interest are in the range of $10^{5} \mathrm{rad} / \mathrm{s}$.

In the calculation of lightning generated induced voltages in power lines it is the first few microseconds, i.e. up to about $10 \mu \mathrm{s}$, of the horizontal field which is mainly of interest. During this time the length of the return stroke channel is not more than 1000 meters. Furthermore, the frequencies which will contribute to the electric field during this time interval are distributed in the range of $10^{7}$ to $10^{5} \mathrm{rad} / \mathrm{s}$. From the results given in figures 2 through 4 we can conclude, therefore, that the surface impedance expression can be used to calculate the horizontal fields generated by return strokes to a high accuracy and for distances larger than or equal to $200 \mathrm{~m}$ the results are almost identical to those calculated by more exact formulation. In the next chapter we will use the surface impedance expression to calculate the horizontal fields generated by lightning return strokes in time domain and, since these are almost identical to the results given by exact formulation, it is reasonable to assume that they are a faithful representation of the horizontal fields generated by lightning.

\section{HORIZONTAL FIELDS GENERATED BY RETURN STROKES}

Let $B_{\mathrm{p}}(\mathrm{t})$ and $E_{\mathrm{h}}(\mathrm{t})$ are the horizontal magnetic field and the horizontal electric field generated by a lightning return stroke over finitely conducting earth. Let $B_{p}(j w)$ and $E_{h}(j w)$ are the corresponding field components in the frequency domain. Then, according to the surface impedance expression

$E_{h}(j \omega) / B_{p}(j \omega)=c /\left(\varepsilon+\sigma / j \omega \varepsilon_{0}\right)^{0.5}$

Performing the inverse Laplace transformation of the above equation, the horizontal field in time domain can be written as

$E_{\mathrm{h}}(\mathrm{t})=\int_{0}^{\mathrm{t}} B_{\mathrm{p}}(\mathrm{t}-\tau) \mathrm{S}(\tau) \mathrm{d} \tau$

where

$S(t)=\left(c / E^{0.5}\right) \zeta e^{-\zeta t}\left[I_{1}(\zeta t)-I_{0}(\zeta t)\right]$

and $\zeta=\varepsilon_{0} \varepsilon / \sigma$. Furthermore, $I_{0}(\zeta t)$ and $I_{1}(\zeta t)$ are the modified Bessel functions of order zero and one respectively with argument $\zeta_{\mathrm{t}}$. A similar procedure can be used to calculate the horizontal field from the wavetilt expression. 
In order to calculate the horizontal field from the above equation it is necessary to know the magnetic field generated by return strokes at a given distance over finitely conducting ground. This in turn requires the knowledge concerning the temporal and spatial variation of the return stroke current along the return stroke channel. Recently, Cooray [10] introduced a new return stroke model which is capable of generating the temporal and spatial variation of the return stroke current along the return stroke channel. The model calculations are in good agreement with the hitherto known properties of the temporal and spatial variation of the return stroke current, spatial variation of the return stroke velocity and the lightning generated electromagnetic fields. In the results to be presented below the electric and magnetic fields generated by lightning return strokes at a given point are calculated by using the model of Cooray [10]. The calculations are performed for a return stroke with a peak current of $10 \mathrm{kA}$ at ground level.

The first $5 \mu$ s of the vertical electric field and the horizontal magnetic field at different distances, when the ground is perfectly conducting are shown in figure 5 . The resulting field components when the ground is finitely conducting are shown in figure 6. Let us denote these field components (i.e. those calculated over finitely conducting ground) as $E_{z}(t)$ and $B_{p}(t)$ respectively. In these calculations the effects of the finitely conducting ground on the electromagnetic fields were incorporated as described in Cooray and Lundquist [11] and Cooray [12]. Note how the risetime of the field components increases with increasing distance. The reason for this is the attenuation of the high frequency components in the electromagnetic fields when they propagate along the finitely conducting earth. The horizontal electric field was then calculated by inserting $B_{\mathrm{p}}(\mathrm{t})$ in equation (7). The results of the calculations are presented in figures 7 and 8 (solid line). As we have mentioned earlier, it is reasonable to assume that they are a faithful representation of the horizontal fields generated by lightning. For comparison purposes the horizontal field calculated by using the wavetilt expression is also given in each figure (dashed line).

First, note that the peak value of the horizontal field, for $\rho=0.001 \mathrm{~S} / \mathrm{m}$, falls off much faster with distance than that of the inverse distance dependence (figure 8). The reason for this is the attenuation of the high frequency components in the magnetic field due to propagation over finitely conducting earth. This also shows the importance of including the propagation effects in the magnetic field which is used to calculate the horizontal field. For example, if we use the magnetic field calculated over perfectly conducting earth (i.e. figure 5) in (7) to calculate the horizontal field the resulting peak amplitude of the horizontal field would be significantly larger than the correct value. For example, in the case of $D=2000 \mathrm{~m}$ and $\rho=0.001 \mathrm{~S} / \mathrm{m}$ the difference will be about $100 \%$. Second, observe the narrow initial peak in the horizontal fields. The higher the conductivity the narrower the initial peak. For example at $200 \mathrm{~m}$ and for $0.01 \mathrm{~S} / \mathrm{m}$ the half width of the initial peak of the horizontal field is about $0.3 \mu$ s (figure 7 ). As shown by Cooray and DeLa Rosa [2] these narrow initial peaks are responsible for the narrow initial peaks measured in induced voltages in power lines. Third, observe that, for a given distance, the peak amplitude of the horizontal field increases with decreasing conductivity. Now let us compare these waveforms with the horizontal fields calculated by using the wavetilt expression. Note that the difference between the two waveforms is larger at small distances than at long distances. For a given distance and conductivity the the error associated with the horizontal field calculated from the wavetilt expression increases with increasing time. For example at $200 \mathrm{~m}$ and for conductivity 0.01 the difference is about $200 \%$ at $5 \mu \mathrm{s}$. Note that even at $1000 \mathrm{~m}$ from the lightning return stroke the horizontal field calculated by the wavetilt expression could be in error by about $50 \%$. 
The results presented in this paper show that the horizontal field generated by return strokes over finitely conducting earth can be calculated to a high accuracy by using either the measured or the calculated horizontal magnetic field in the expression for the surface impedance. The method is suitable to calculate horizontal fields for distances down to about $200 \mathrm{~m}$ from the return strokes. The use of wavetilt expression to calculate the horizontal field can result in large errors specially when the return stroke is within about a kilometer from the point of observation.

\section{ACKNOWLEDGEMENTS}

The research work reported here was supported by a grant (No. E-EG 1448-303) to the author from the Swedish Natural Science Research Council.

\section{REFERENCES}

[1] Master, M. J. and M. A. Uman, Lightning-induced voltages on power line: Theory, IEEE Trans., PAS-103, pp. 2502-2518, September, 1984.

[2] Cooary, V. and De La Rosa, F., Shapes and amplitudes of the initial peaks of lightning induced voltage in power lines over finitely conducting earth: Theory and comparison with experiment, IEEE Trans., vol. AP. 34, pp. 88-92, January, 1986.

[3] Rubinstein, M., M. A. Uman, E. M. Thomson and Pedro Medelius, Voltages induced on a test line by artificially initiated lightning at close range: Measurements and Theory, Proceedings of the International Conference on Lightning Protection, Interlaken, Switzerland, 1990.

[4] Sommerfeld, Uber die Ausbreitung der Wellen in der drahtlosen Telegraphie, Ann. Phys. $28,665,1909$.

[5] Norton, K, A., Propagation of radio waves over the surface of the earth and in the upper atmosphere, II, Proc. IEEE, 25, pp. 1203-1236, 1937.

[6] Banos, A., Dipole radiation in the presence of a conducting half-space, Pergamon press, 1966.

[7] Sakar, T. K., Analysis of radiation by arrays of vertical wire antennas over imperfect ground (reflection coefficient method), IEEE Trans., vol. AP.23, September, 1975.

[8] Bannister, P. R., Extension of finitely conducting Earth-image-theory results to any range, Technical Report 6885, Naval Under Water Systems Center, January, 1984.

[9] Zeddam, Z. and P. Degauque, Current and Voltage induced on telecommunication cables by a lightning stroke, Electromagnetics, vol. 8, pp. 171-211, 1988.

[10] Cooray, V., A return stroke model, Proceedings of the 1989 International Conference on Lightning and Static Electricity, 1A.3.1-1A.3.9, University of Bath, United Kingdom, September, 1989.

[11] Cooray, V., and S. Lundquist, Effects of propagation on the risetimes and the initial peaks of the radiation fields from return strokes, Radio Sci., vol. 18, pp. 409-415, 1983.

[12] Cooray, V., Effects of propagation on the return stroke radiation fields, Radio Sci., vol. 22, pp. 752-768, 1987. 


\section{GLOSSARY OF SYMBOLS}

\begin{tabular}{|c|c|}
\hline $\begin{array}{l}\mathbf{R}_{0} \\
\mathbf{R}_{1} \\
\mathbf{R}_{2}\end{array}$ & $\begin{array}{l}{\left[D^{2}+(z-h)^{2}\right]^{0.5} \text { (meters) }} \\
{\left[D^{2}+(z+h)^{2}\right]^{0.5} \text { (meters) }} \\
{\left[D^{2}+(d+z-h)^{2}\right]^{0.5} \text { (meters) }}\end{array}$ \\
\hline$\psi_{0}$ & $\tan ^{-1}\left(\frac{z-h}{D}\right)$ \\
\hline$\psi_{1}$ & $\tan ^{-1}\left(\frac{z+h}{D}\right)$ \\
\hline$\Psi_{2}$ & $\tan ^{-1}\left(\frac{d+z-h}{D}\right)$ \\
\hline $\begin{array}{l}\mathrm{z} \\
\mathrm{D} \\
\mathrm{h}\end{array}$ & $\begin{array}{l}\text { Height of the point of observation with respect to earth's surface } \\
\text { Horizontal distance between the dipole and the point of observation } \\
\text { Height of the dipole with respect to earth's surface }\end{array}$ \\
\hline$\sigma$ & Conductivity of the earth \\
\hline$\varepsilon$ & Relative dielectric constant of the earth \\
\hline$\varepsilon_{0}$ & Permittivity of free space \\
\hline$\mu_{0}$ & Permeability of free space \\
\hline $\begin{array}{l}\omega \\
j\end{array}$ & $\begin{array}{l}\text { Angular frequency (radians/s) } \\
(-1)^{0.5}\end{array}$ \\
\hline$\gamma_{0}$ & $j \omega / c$ \\
\hline$\gamma_{1}$ & {$\left[j \omega \mu_{0} \sigma-\omega^{2} \mu_{0} \varepsilon \varepsilon_{0}\right]^{0.5}$} \\
\hline $\mathrm{d}$ & $2 / \gamma_{1}$ \\
\hline$\Delta$ & $\gamma_{0} / \gamma_{1}$ \\
\hline $\begin{array}{l}\mathrm{n} \\
\mathrm{F} \\
\mathrm{M} \\
\mathrm{c}\end{array}$ & 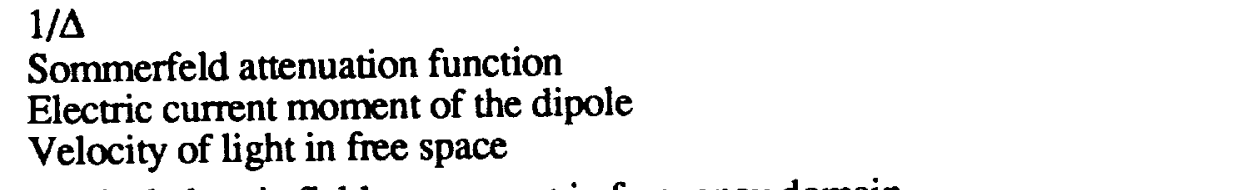 \\
\hline$E_{z}(j \omega)$ & Vertical electric field component in frequency domain \\
\hline$E_{h}(j \omega)$ & Horizontal electric field component in frequency domain \\
\hline $\begin{array}{l}B_{p}(j \omega) \\
E_{h w} \\
E_{h s} \\
E_{h}(t) \\
B_{p}(t)\end{array}$ & $\begin{array}{l}\text { Horizontal magnetic field component in frequency domain } \\
\text { Horizontal electric field component calculated from the wavetilt } \\
\text { Horizontal electric field component calculated from the surface impedance } \\
\text { Horizontal electric field in time domain generated by lightning stroke } \\
\text { Horizontal magnetic field in time domain generated by lightning stroke }\end{array}$ \\
\hline
\end{tabular}




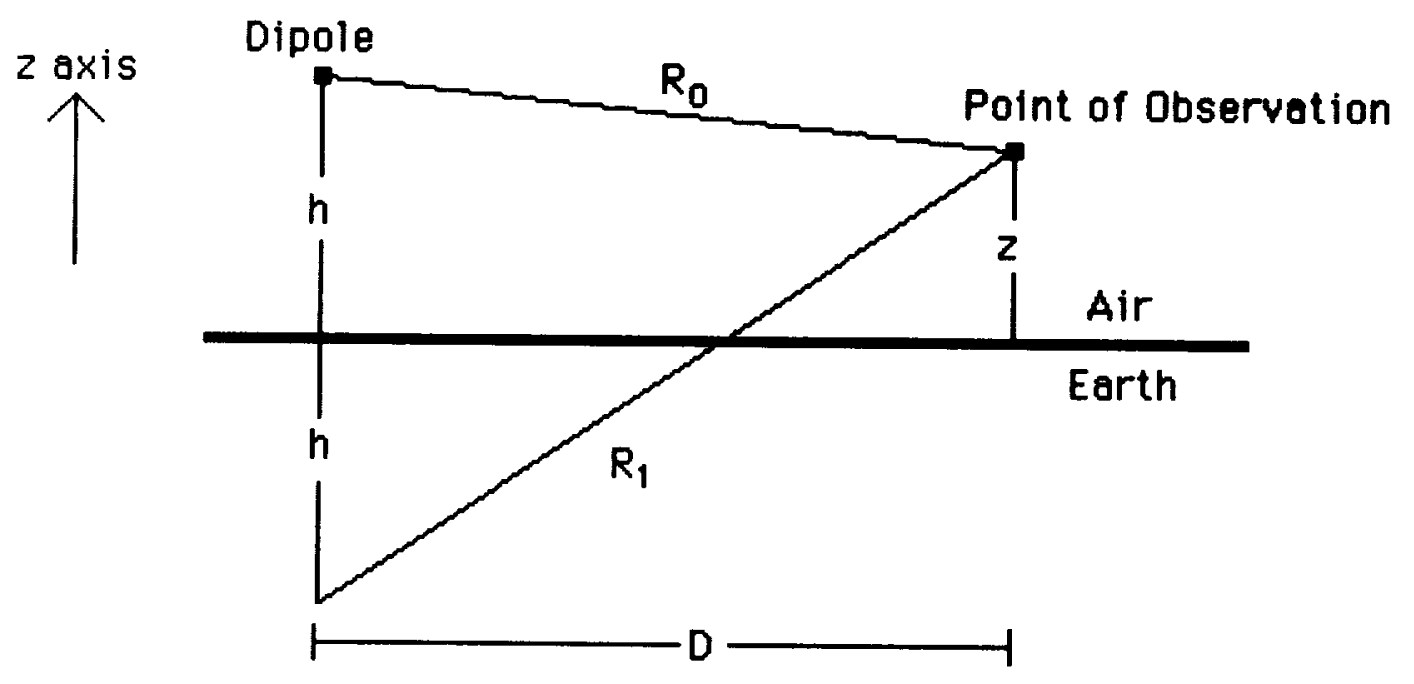

Fig. 1 Geometry relevant to the problem under consideration.
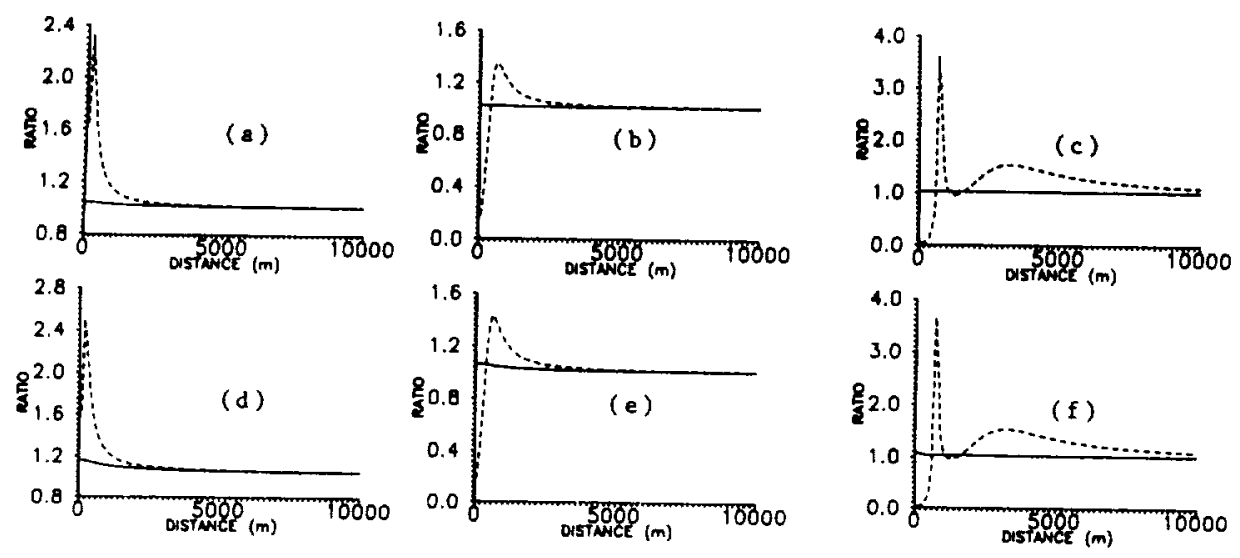

Fig.2 The variation of the ratios $\left|\mathrm{E}_{\mathrm{hs}} / \mathrm{E}_{\mathrm{h}}\right|$ (solid line) and $\left|\mathrm{E}_{\mathrm{hw}} / \mathrm{E}_{\mathrm{h}}\right|$ (dashed line) as a function of distance for (a) $\omega=10^{7} \mathrm{rad} / \mathrm{s}$ and $\sigma=0.01 \mathrm{~S} / \mathrm{m}$ (b) $\omega=10^{6} \mathrm{rad} / \mathrm{s}$ and $\sigma=0.01$ $\mathrm{S} / \mathrm{m}$ (c) $\omega=10^{5} \mathrm{rad} / \mathrm{s}$ and $\sigma=0.01 \mathrm{~S} / \mathrm{m}$ (d) $\omega=10^{7} \mathrm{rad} / \mathrm{s}$ and $\sigma=0.001 \mathrm{~S} / \mathrm{m} \mathrm{(e)} \omega=10^{6}$ $\mathrm{rad} / \mathrm{s}$ and $\sigma=0.001 \mathrm{~S} / \mathrm{m}$ and $(\mathrm{f}) \omega=10^{5} \mathrm{rad} / \mathrm{s}$ and $\mathrm{s}=0.001 \mathrm{~S} / \mathrm{m}$. The dipole is situated at ground level i.e. $h=0$. 

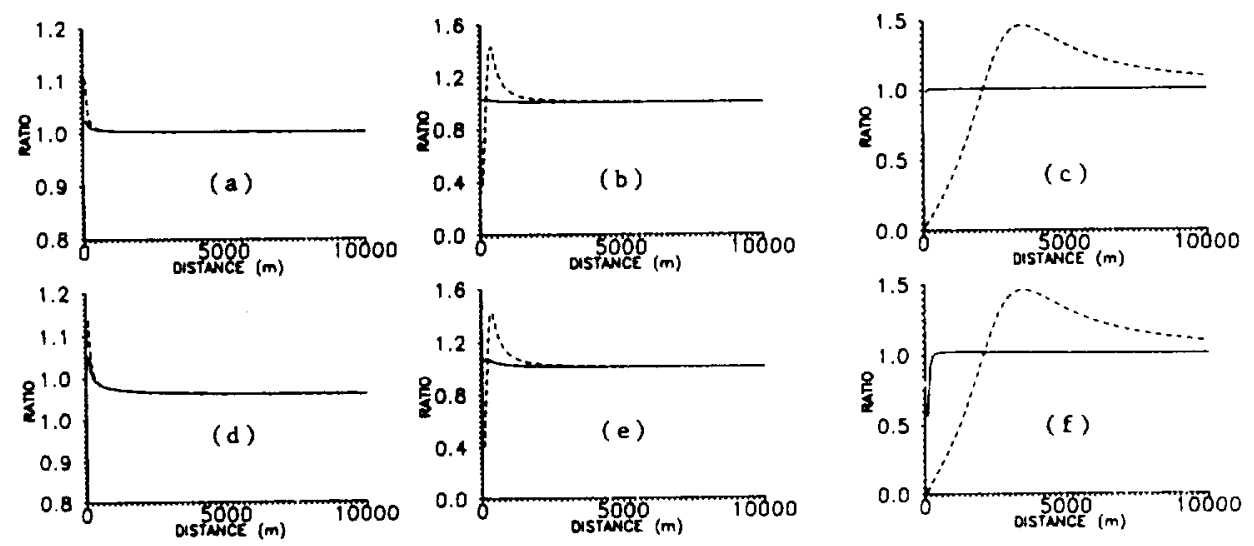

Fig.3 Same as in figure 2 except that the dipole is at $500 \mathrm{~m}$ from ground level.
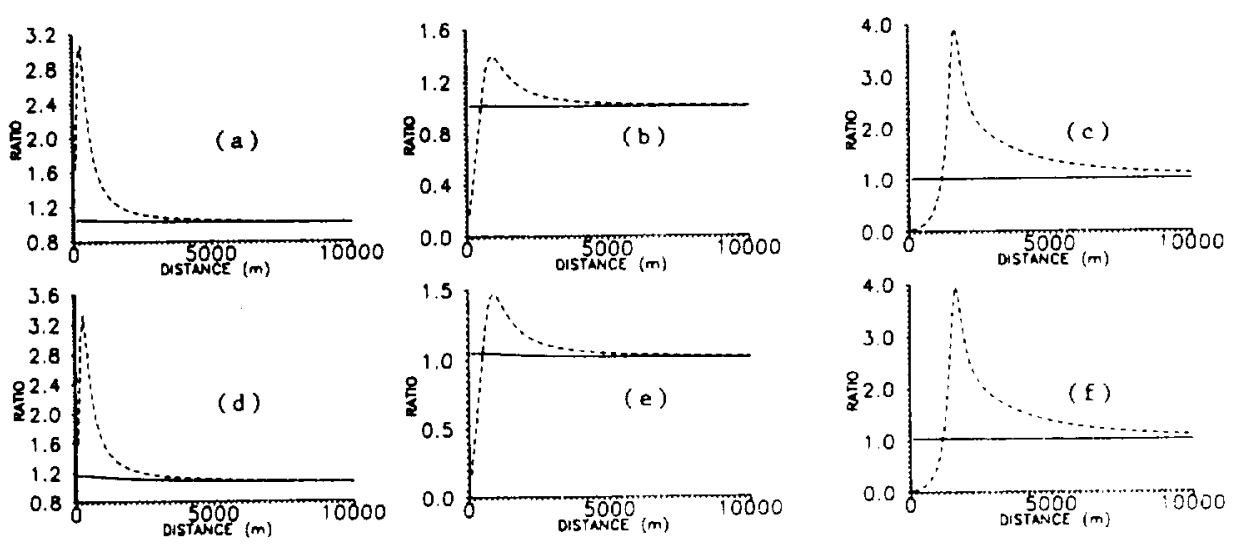

Fig.4 Same as in figure 2 except that the dipole is at $1000 \mathrm{~m}$ from ground level. 

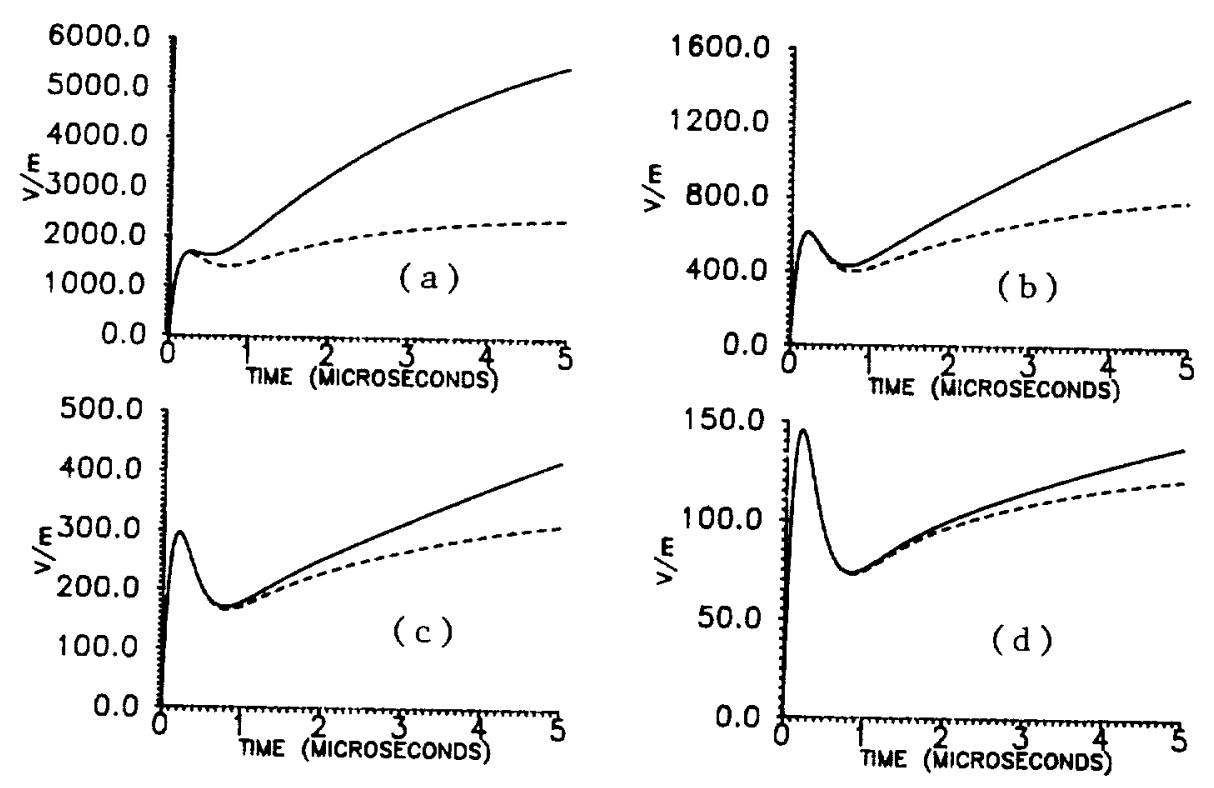

Fig.5 Vertical electric field (solid line) and the horizontal magnetic field $x \mathrm{c}$ (dashed line) generated by a return stroke over perfectly conducting ground, as calculated by using the model of Cooray (1989). The peak current in the return stroke is $10 \mathrm{kA}$. The distances to the point of observations are the following: (a) $200 \mathrm{~m}$, (b) $500 \mathrm{~m}$, (c) $1000 \mathrm{~m}$ and (d) $2000 \mathrm{~m}$.
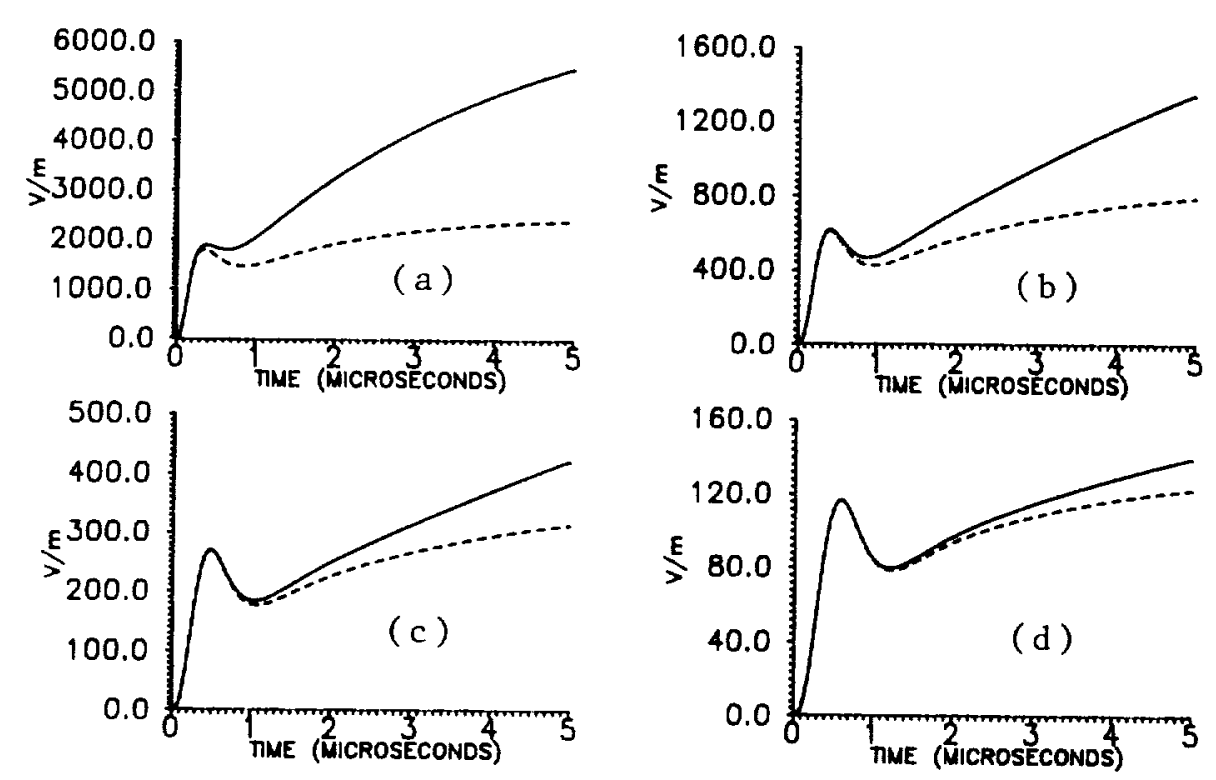

Fig.6 Same as in figure 5 except that the conductivity of the ground (i.e. $\sigma$ ) is $0.001 \mathrm{~S} / \mathrm{m}$. 

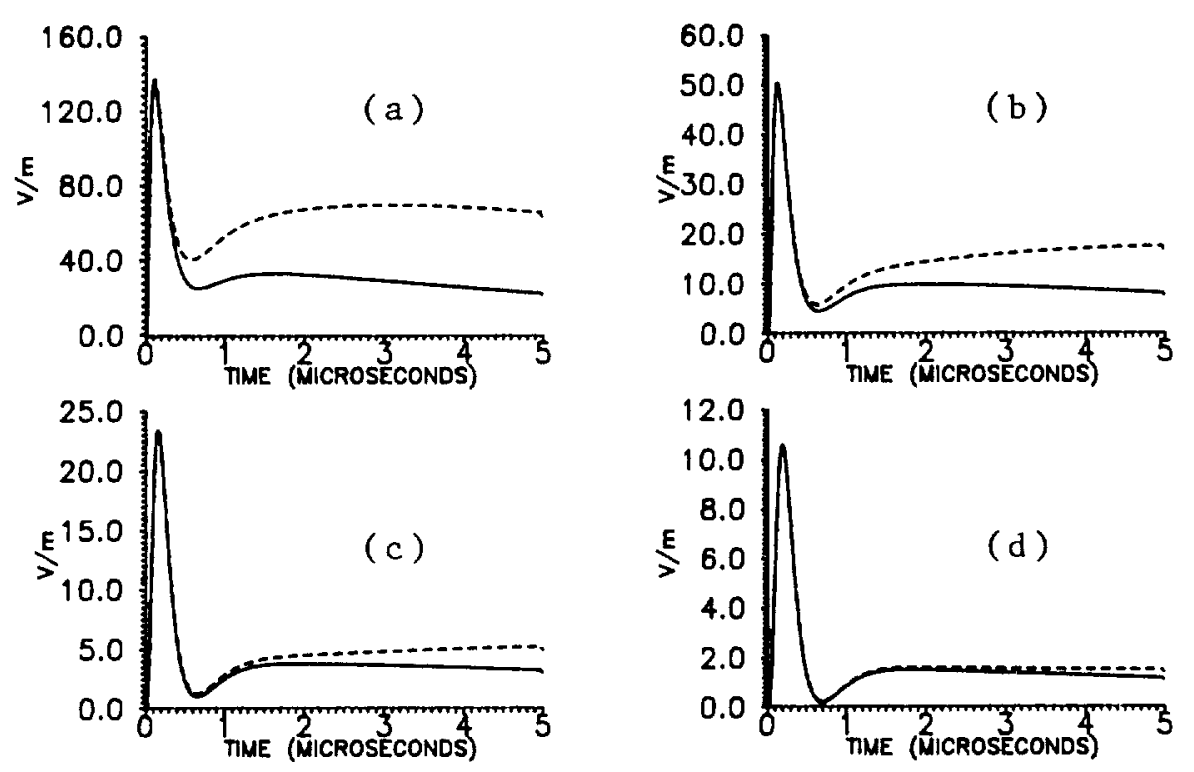

Fig.7 Horizontal field generated by a return stroke at different distances when the conductivity of the ground is $0.01 \mathrm{~S} / \mathrm{m}$ (solid line). The peak current in the return stroke is 10 $\mathrm{kA}$. The distances to the point of observations are the following: (a) $200 \mathrm{~m}$, (b) $500 \mathrm{~m}$, (c) $1000 \mathrm{~m}$ and (d) $2000 \mathrm{~m}$. The horizontal fields calculated by using the wavetilt expression are drawn by dashed lines.
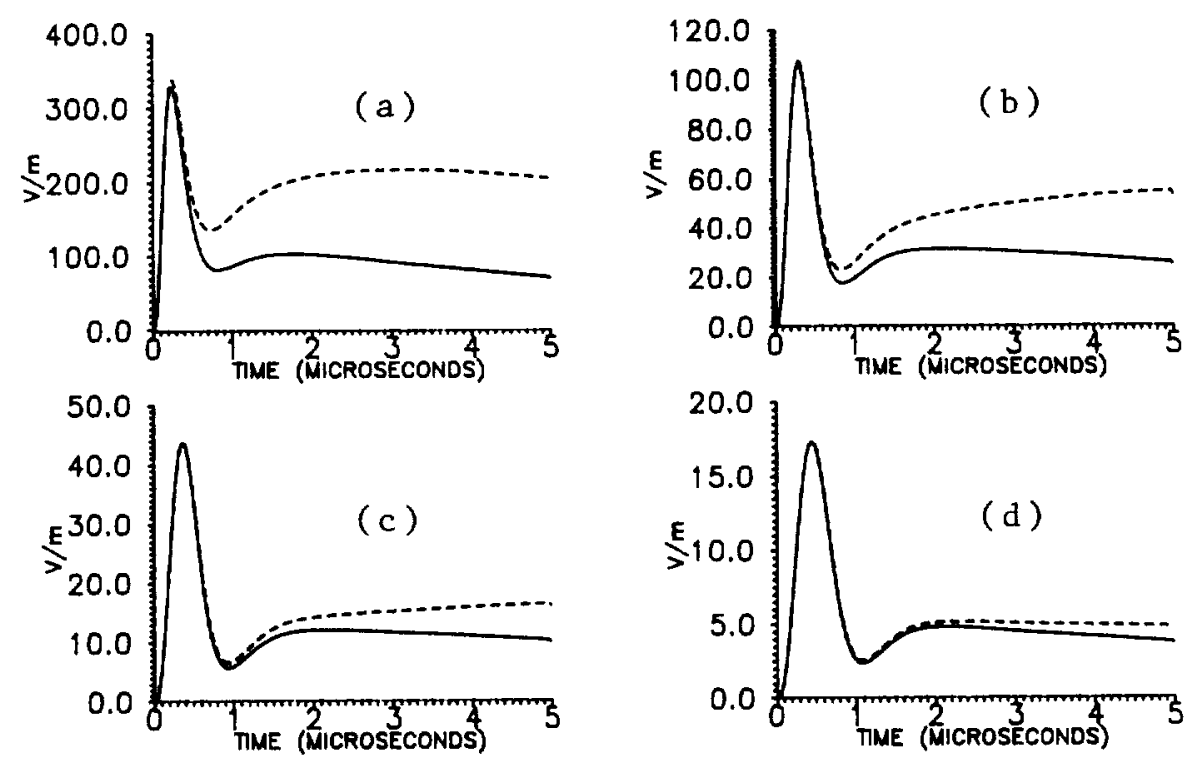

Fig.8 Same as in figure 7 except that the conductivity of the ground is $0.001 \mathrm{~S} / \mathrm{m}$. 